\title{
Delusion formation and reasoning biases in those at clinical high risk for psychosis
}

\author{
M. R. BROOME, L. C. JOHNS, I. VALLI, J. B. WOOLLEY, P. TABRAHAM, \\ C. BRETT, L. VALMAGGIA, E. PETERS, P. A. GARETY and P. K. McGUIRE
}

\author{
Background Cognitive models \\ propose that faulty appraisal of anomalous \\ experiences is critical in developing \\ psychosis, particularly delusions. A data \\ gathering bias may be fundamental to \\ abnormal appraisal.
}

Aims To examine whether there is a data gathering bias in people at high risk of developing psychosis.

\begin{abstract}
Method Individuals with an at-risk mental state $(n=35)$ were compared with a matched group of healthy volunteers $(n=23)$. Participants were tested using a modified version of the 'beads' reasoning task with different levels of task difficulty.
\end{abstract}

Results When task demands were high, the at-risk group made judgements on the basis of less information than the control group $(P<0.05)$. Within both groups, jumping to conclusions was directly correlated with the severity of abnormal beliefs and intolerance of uncertainty $(P<0.05)$. In the at-risk group it was also associated with impaired working memory $(P<0.05)$, whereas in the control group poor working memory was associated with a more conservative response style $(P<0.05)$.

Conclusions People with an at-risk mental state display a jumping to conclusions reasoning style, associated with impaired working memory and intolerance of uncertainty. This may underlie a tendency to develop abnormal beliefs and a vulnerability to psychosis.

Declaration of interest None. Funding detailed in Acknowledgements.
Both cognitive and neurobiological models propose that a critical factor in the development of psychosis is the faulty appraisal or interpretation of anomalous experiences or events (Garety \& Freeman, 1999; Freeman et al, 2002; Kapur, 2003; Broome et al, 2005a; Freeman, 2007; Garety et al, 2007). One aspect of appraisal, reasoning, can be studied using the 'Beads' paradigm, in which the subject is shown a series of different coloured beads and is required to guess which of two jars they have been drawn from. Patients with psychotic disorders require fewer beads to be drawn before they are sure of their source than controls, yet are not any less accurate. This 'jumping to conclusions' response style has been interpreted as reflecting a data gathering reasoning bias. As well as in patients with psychosis, jumping to conclusions has been described in volunteers with high levels of delusional ideation (Linney et al, 1998) and the relatives of patients with psychosis (Van Dael et al, 2006). In all these groups jumping to conclusions has been particularly associated with the intensity of delusional ideation (Linney et al, 1998; Garety \& Freeman, 1999; Peters et al, 1999; Garety et al, 2005; Van Dael et al, 2006). The mechanisms underlying jumping to conclusions are unclear, but it has been investigated in relation to an impaired ability to hold information online (Dudley et al, $1997 a$ ) and to an inability to tolerate ambiguity (Colbert $\&$ Peters, 2002).

We tested the hypothesis that participants with an at-risk mental state would be more likely to jump to conclusions than controls. Secondary predictions were that the tendency to jump to conclusions would be associated with impaired working memory and an intolerance of uncertainty, and would predict the severity of abnormal beliefs.

\section{METHOD}

People with 'prodromal' symptoms of psychosis have a $25-40 \%$ risk of developing a psychotic disorder in the next 12 months (Yung et al, 1998, 2003; Miller et al, 2003) and thus have an at-risk mental state. Individuals with an at-risk mental state were recruited from Outreach and Support in South London (OASIS) (Broome et al, 2005b). All met PACE criteria (Yung et al, 2003) for the at-risk mental state: an individual can meet criteria for this state in one or more of three ways. First, a recent decline in function coupled with either schizotypal personality disorder or a firstdegree relative with psychosis. Second, 'attenuated' positive symptoms and third, a brief psychotic episode of less than 1 week's duration that resolves without antipsychotic medication. Each participant was assessed by two experienced clinicians using the Comprehensive Assessment for the at-risk mental state (CAARMS; Yung et al, 2003), and the diagnosis was confirmed at a consensus meeting with the clinical team. None of the participants had been exposed to antipsychotic medication.

Healthy volunteers $(n=23)$ were recruited via advertisements in the local media.

All participants lived in the same borough of London (Lambeth), were native speakers of English and were right-handed. The groups were matched on sociodemographic variables. Participants were excluded if there was a history of neurological disorder or if they met DSM-IV criteria for a substance misuse or dependence disorder.

\section{Assessment of psychopathology}

Psychopathology was assessed using the CAARMS, the Peters' Delusions Inventory (PDI; Peters et al, 1999), the Positive and Negative Symptom Scale (PANSS; Kay et al, 1987) and the delusion subscale of the Scale for the Assessment of Positive Symptoms (SAPS; Andreason, 1984).

\section{Reasoning task}

The tendency for participants to 'jump to conclusions' was examined using a modified version of the 'beads' reasoning task (Garety \& Freeman, 1999; Freeman, 2007). In the beads task, participants are shown two jars of coloured beads, informed of the relative proportions of beads in each, then told that they will be shown a series of beads drawn from one of the jars. They are then asked, on the basis of the observed sequence, to judge which jar is the source of the beads, and to be 'as certain 
as possible', but it is never possible to be completely certain as to which jar the beads have been drawn from (Huq et al, 1988; Garety \& Freeman, 1999).

As in the classical version of the paradigm, participants in our study were informed that a series of beads would be drawn from one of two jars containing beads of two colours in the ratios $85: 15$ and 15:85. They were instructed to monitor the colours of successively drawn beads until they were as certain as they could be as to which of the jars the beads were being drawn from. A pseudo-random predetermined list was used to determine the colour of bead shown. Beads were presented on a computer screen at $1 \mathrm{~s}$ intervals, with participants responding via a button press. The modified version involved 3 conditions: (a) 2 jars with bead ratios of 85:15, (b) 2 jars with 60:40 and (c) 3 jars with 44:28:28. Participants were asked to indicate which jar the beads were being drawn from when they were 'as certain as possible'. Real jars of beads in the appropriate ratios and colours were shown to the subjects when the task was being explained beforehand.

\section{Working memory}

The ability to hold information about bead colour online was assessed using an adaptation of the digit span task that used a string of different coloured beads (between 5 and 9; as in the beads task) rather than numbers. Participants were presented with 5 different length strings of coloured beads, 2 trials of each, using a laptop. Beads were presented at $1 \mathrm{~s}$ intervals and after presentation participants were asked to recall the order of the colour in which beads were presented. Longest span of beads and total errors were recorded.

\section{Tolerance of uncertainty}

Tolerance of uncertainty was evaluated using the Freeston Intolerance of Uncertainty scale (Freeston et al, 1994). This questionnaire is a 27 -item Likert scale and was designed to generate a single summary score and cover a wide range of concepts, but factor analyses of the scale identified constructs covering 'behavioural attempts to control the future and avoid uncertainty, inhibition of action, emotional reactions such as frustration and stress, and cognitive interpretations that being uncertain reflects badly on a person' (Freeston et al, 1994; p. 799). Intolerance of uncertainty is conceptualised as a manifestation of basic dysfunctional (trait) schema that may in turn guide information processing and appraisal. It can generate and maintain anxiety in ambiguous situations both through facilitating the perception of difficulties where none exist, and where difficulties do exist, lead to inefficient responses to them.

\section{IQ}

Premorbid and current intellectual function was estimated using the National Adult Reading Test (NART; Nelson, 1991) and the Quick test (Ammons \& Ammons, 1962).

\section{RESULTS}

\section{Descriptive statistics and comparisons of means}

Controls and participants at risk were matched on a measure of current IQ as measured by the Quick test: controls 104.7 (s.d.=13.4); at-risk mental state 100.1 (s.d.=9.8) and on age: controls 24.9 years $($ s.d. $=3.0)$; at-risk mental state 24.2 years, (s.d.=4.3), but differed on premorbid IQ as estimated by the NART: controls 110.9 (s.d.=7.3) and at-risk mental state 102.3 (s.d.=10.6). Participants with at-risk mental state had a mean PANSS total score of 52.1 (s.d.=14.5), and the positive symptom sub-scale was 12.2 (s.d.=3.9). For the delusion sub-scale of the SAPS, the at-risk mental state group had a mean score of 3.9 (s.d. $=4.0$ ).

\section{Beads task}

There was no significant difference in performance of the participants with at-risk mental state subjects in comparison to the control group on the classical (or 'easy') version of the beads task: the mean number of beads viewed by participants with at-risk mental state before they responded was 7.4 compared to 6.4 for controls (Table 1, Fig. 1). However, on both of the harder versions of the task (60:40 and 44:28:28) the at-risk mental state group drew fewer beads than controls before responding. For the intermediate version of the task the mean number of beads viewed by participants with at-risk mental state before they responded was 8.5 , but for controls was $13.4(P<0.001)$. On the hard version of the task, those with at-risk mental state viewed 12.5 beads and controls 17.5
$(P=0.012)$. (Fig. 1, Table 1). Both these differences in performance remained significant after co-varying for differences in NART score.

\section{Delusional ideation}

There were highly significant differences between the at-risk mental state and control groups on the total PDI score, and on each of the distress, preoccupation and conviction sub-scales. On all these measures the at-risk mental state group scored higher than controls. (Table 2).

\section{Working memory}

The at-risk mental state group had a significantly shorter span for correct responses than controls on the beads span task, and made significantly more errors (Table 2 ).

\section{Intolerance of uncertainty}

The at-risk mental state group had significantly higher ratings on the Freeston Intolerance of Uncertainty scale than controls (Table 2).

\section{Correlations with beads task performance \\ PDI scores}

For both groups on both the intermediate and hard conditions of the beads task there was an inverse relationship between the number of beads viewed before the response and scores on the PDI and each of its sub-scales. These were statistically significant for the total PDI score and all three PDI sub-scales on intermediate $(60: 40)$ version of the task and evident as trends for the hard version (Table 3). The strongest and most significant correlation was with scores on the conviction sub-scale of the PDI. There were no significant correlations between performance on the easy $(85: 15)$ version and any of the PDI measures.

\section{Intolerance of uncertainty}

In both groups the number of beads viewed was inversely related to the Intolerance of Uncertainty score, with a significant correlation on the intermediate version and a trend on the hard version of the task (Table 3).

\section{Symptom scores}

Within the at-risk mental state group there were no significant or trend correlations 
Table I Beads task performance by task difficulty and group

\begin{tabular}{lccc}
\hline & $\begin{array}{c}\text { Controls } \\
(n=23)\end{array}$ & $\begin{array}{c}\text { Participants with at-risk } \\
\text { mental state }(n=35)\end{array}$ & $P$ \\
\cline { 2 - 3 } & \multicolumn{2}{c}{ mean (s.d.) } \\
\hline Easy - 85:15 & $6.4(3.3)$ & $7.4(4.4)$ & NS \\
Intermediate - 60:40 & $13.4(5.9)$ & $8.5(3.7)$ & $<0.001$ \\
Hard - 44:28:28 & $17.5(8.1)$ & $12.5(5.7)$ & 0.012 \\
\hline
\end{tabular}

between task performance (on any version) and either the PANSS (both total score and positive sub-scale), or the delusion subscale of the SAPS.

\section{Working memory}

There were no significant correlations between performance on the beads task and the bead span across both groups of participants combined, but there were correlations within each group. In controls the number of beads drawn in all versions of the task was directly correlated with the number of errors on the bead span task, although this only reached significance on the intermediate version (Table 4). Conversely, in the at-risk mental state group there was a negative correlation between beads viewed and errors on the bead span task. Again this was only significant on the intermediate version of the task (Table 4).

\section{DISCUSSION}

\section{Jumping to conclusions}

Our first hypothesis was confirmed in that the at-risk mental state group demonstrated a jumping to conclusions style of thinking, although this was evident when the task demands were increased by making the proportions of the respective beads more similar $(60: 40)$ or by introducing a third

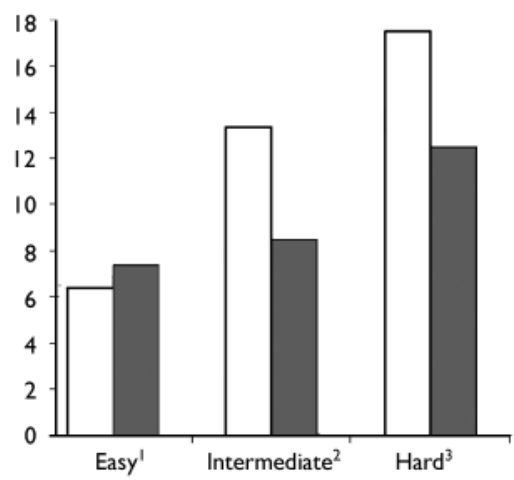

Fig. I Draws to decision by task difficulty and group. $\square$, at-risk mental state group; $\square$, controls. colour of bead (44:28:28), rather than during the 'classic' (85:15) version. Our findings are consistent with those of Linney $e t$ al (Linney et al, 1998), who reported that non-clinical individuals with high scores on the PDI showed a jumping to conclusions response style, although in that case differences were evident using the classical version of the task. Our findings are also consistent with work demonstrating an association between a jumping to conclusions bias and both psychosis liability (as indexed by family history of psychosis and/or psychotic experiences) and delusional ideation (Linney et al, 1998; Peters et al, 1999; Van Dael et al, 2006). While the jumping to conclusions style of thinking in subjects at very high risk of psychosis has not been examined before, the at-risk mental state has been associated with deficits in working and episodic memory and executive functions (Wood et al, 2003; Brewer et al, 2005; Broome et al, 2007, submitted further information available from author). Further, recent work on the bias in groups at differing liability to psychotic illness, has revealed a dose-response relationship between the jumping to conclusions bias and both delusional ideation and psychosis liability (Van Dael et al, 2006); span leading to the suggestion that the jumping to conclusions bias is both a trait and state variable in the risk and progression of psychosis.

The finding that jumping to conclusions bias is present in those at high risk of psychosis is consistent with cognitive models that suggest that the faulty appraisal of anomalous experiences plays a fundamental role in the development of the disorder (Broome et al, 2005a; Garety et al, 2005, 2007). While 'jumping to conclusions' has consistently been found in patients with established psychosis (Garety \& Freeman, 1999), its presence in individuals at very high risk of the disorder suggests that the presence of this impairment may influence whether an individual who is experiencing psychotic symptoms progresses to frank psychosis. Van Dael and colleagues (2006) suggest that the jumping to conclusions bias, as well as being a trait vulnerability, may have a state component and one would expect such a bias to increase, and be detectable at lower levels of task demand, as at-risk mental state participants made the transition to psychosis. Conversely, those in whom the at-risk mental state remitted may demonstrate an attenuation of the jumping to conclusions bias. This could be tested in a longitudinal study of subjects with an at-risk mental state.

\section{Jumping to conclusions and the severity of abnormal beliefs}

Consistent with previous studies (Garety et al, 2005), we found that jumping to conclusions response style was associated with the severity of abnormal beliefs, as indexed by the PDI. However, this finding was evident across all subjects, rather than

Table 2 Group comparison for delusional ideation, intolerance of uncertainty, Bead span and errors on Bead

\begin{tabular}{lccc}
\hline & $\begin{array}{c}\text { Controls } \\
(n=23)\end{array}$ & $\begin{array}{c}\text { Participants with at-risk } \\
\text { mental state }(n=35)\end{array}$ & $P$ \\
\cline { 2 - 4 } & \multicolumn{2}{c}{ mean (s.d.) } \\
\hline PDI total & $29.2(26.0)$ & $99.0(57.0)$ & 0.000 \\
PDI distress & $9.0(10.3)$ & $34.4(23.0)$ & 0.000 \\
PDI preoccupation & $8.9(7.7)$ & $32.4(20.7)$ & 0.000 \\
PDI conviction & $11.5(9.1)$ & $32.1(16.2)$ & 0.000 \\
Intolerance of Uncertainty & $58.3(15.3)$ & $79.8(22.8)$ & 0.000 \\
Maximum bead span & $7.8(0.6)$ & $6.6(1.0)$ & 0.000 \\
Total errors on bead span & $3.7(3.4)$ & $10.2(8.9)$ & 0.001 \\
\hline
\end{tabular}


Table 3 Correlations with performance (beads drawn) on the intermediate' and hard ${ }^{2}$ versions of the bead task across all groups (i.e. at-risk mental state and controls). ${ }^{3}$

\begin{tabular}{|c|c|c|c|c|c|}
\hline & PDI total & PDI distress & PDI preoccupation & PDI conviction & Intolerance of Uncertainty \\
\hline \multicolumn{6}{|c|}{ Pearson correlation, $r(P)$} \\
\hline Beads - intermediate' & $-0.331(0.019)$ & $-0.275(0.05)$ & $-0.325(0.021)$ & $-0.375(0.007)$ & $-0.322(0.019)$ \\
\hline Beads - hard ${ }^{2}$ & $-0.237(0.097)$ & $-0.196(0.17 I)$ & $-0.244(0.88)$ & $-0.258(0.07 I)$ & $-0.242(0.08 \mathrm{I})$ \\
\hline
\end{tabular}

PDI, Peters' Delusions Inventory.

I. Intermediate refers to the $60: 40$ condition.

2. Hard refers to the $44: 28: 28$ condition.

3. Negative correlation with beads drawn implies positive correlation with data gathering bias.

being specific to the at-risk mental state group. This is further evidence that rather than being a correlate of frank psychosis, the tendency to jump to conclusions may vary continuously across clinical categories. The association with delusions does not seem simply to reflect jumping to conclusions in those subjects with the most psychotic symptoms, as there was no correlation with either the total PANSS score or the positive symptom sub-scale, or with the SAPS delusion sub-scale score. A specific association with delusions is consistent with studies in established psychosis (Garety \& Freeman, 1999) and has face validity, in that of all the psychotic symptoms, abnormal beliefs are the most dependent on the participant's interpretation of his experiences. A parsimonious interpretation of the data would be that jumping to conclusions is a sign of faulty appraisal, which is the basis of delusional beliefs, regardless of whether these are held by an individual with psychosis, at-risk mental state, or without psychosis. According to this model, the worse the jumping to conclusions, the more severe the appraisal problem and the more severe the delusions. Faulty appraisal may not distinguish people with psychotic symptoms from people with a psychotic illness in a categorical way, rather faulty appraisal may underlie the subset of psychotic symptoms that depend on the conscious evaluation of sensory/internal information, i.e. delusional beliefs (and perhaps hallucinations), as opposed to the syndrome of psychosis. Appraisal and jumping to conclusions may be less relevant to psychotic symptoms that are less dependent on the conscious appraisal of experiences, for example formal thought disorder, negative symptoms.

\section{Jumping to conclusions and intolerance of uncertainty}

The basis of the data gathering bias is unclear. One factor may be the decision-making style of the individual. Although jumping to conclusions is not simply related to impulsivity (Dudley et al, 1997a,b), it may be more evident in individuals who find it difficult to tolerate ambiguity (Colbert $\&$ Peters, 2002). The at-risk mental state group scored higher on the Intolerance of Uncertainty scale than controls. Difficulties tolerating uncertainty may thus have contributed to the jumping to conclusions response style in the at-risk mental state group. As with the PDI, there was a significant correlation across both groups between intolerance of uncertainty and the data gathering bias. The group differences in tolerating uncertainty may be related to the relatively high prevalence of personality and neurotic disorder among participants with at-risk mental state (Broome et al, 2005b).

\section{Jumping to conclusions and working memory}

Another factor that could contribute to jumping to conclusions is impaired working memory, with subjects making earlier decisions because of difficulties holding material that would inform their judgement online, although the evidence for this is limited. Consistent with previous studies of working memory in participants with at-risk mental state (Wood et al, 2003; Brewer et al, 2005; Brett et al, 2007, in press), the at-risk mental state group displayed poorer performance on the bead span task than controls. This is consistent with our prediction that impaired working memory would contribute to a jumping to conclusions response style. Moreover jumping to conclusions on one of the harder versions (60:40 bead ratio) of the beads task in the at-risk mental state group was correlated with impaired performance on the beads span task. These data suggest that a difficulty in holding information online may contribute to participants making judgements in which they can never be certain sooner than they might do otherwise. Dudley et al (1997a) did not find an association between jumping to conclusions response style and memory impairments in patients with psychosis, but this may reflect the use of the classical beads task without the more difficult conditions, or the fact being reminded of the beads one has seen does not guarantee that that information is itself able to be utilised in reasoning. In the present study, the effect of memory impairment was divergent in the two groups: in the control group, there was an increased conservatism and caution in those with poorer ability to recall sequences of beads, the opposite to what was evident in the at-risk mental state group. This suggests that controls with poor working memory compensated by seeking more information, or did not find being uncertain how to respond as aversive as the participants at-risk.

In summary, people who are at high risk of psychosis display a jumping to conclusions reasoning style which is associated

Table 4 Pearson correlations with bead span errors by group and task difficulty

\begin{tabular}{lll}
\hline & Controls $(n=23)$ & Participants with at-risk mental state $(n=35)$ \\
\cline { 2 - 3 } & & Pearson correlation $r(P)$ \\
\hline Easy & $0.277(0.213)$ & $-0.143(0.452)$ \\
Intermediate & $0.444(0.039)$ & $-0.562(0.0 .001)$ \\
Hard & $0.237(0.289)$ & $-0.279(0.135)$ \\
\hline
\end{tabular}


with a difficulty in tolerating uncertainty and impaired working memory. A reasoning bias may be a critical factor in the development of clinically significant psychotic phenomena and contribute to the high risk of frank psychosis in this group. More generally, the findings are compatible with data from structural (Pantelis et al, 2003) and functional neuroimaging (Morey et al, 2005; Broome et al, 2007, submitted - further information available from author) and neuropsychological studies (Wood et al, 2003; Brewer et al, 2005 in subjects with an at-risk mental state, which broadly indicate that this group displays abnormalities that are qualitatively similar to those seen in patients with schizophrenia but quantitatively less severe. Approximately one third of those with an at-risk mental state will develop psychosis (Yung et al, 2003; Morrison et al, 2004; Broome et al, 2005a). We are currently in the process of following up our at-risk mental state sample to establish whether task performance predicts development of psychosis subsequent to testing.

\section{ACKNOWLEDGEMENTS}

Special thanks go to all those who took part in the study and the staff of the OASIS team.

The study was supported by the Guy's and St Thomas' Charitable Foundation and the Mental Health Foundation. Modification, piloting of the task, and initial data collection was carried out when M.R.B. was supported by the South London and Maudsley NHS Trust as Maudsley Research Registrar to the Institute of Psychiatry.

\section{REFERENCES}

Ammons, R. \& Ammons, C. (1962) Quick Test. Psychological Test Specialists.

Andreason, N. C. (1984) The Scale for the Assessment of Positive Symptoms (SAPS). University of lowa.

Brewer, W. J., Francey, S. M., Wood, S. J., et al (2005) Memory impairments identified in people at ultra-high risk for psychosis who later develop first-episode psychosis. American Journal of Psychiatry, 162, 7I-78.

Broome, M. R., Woolley, J. B., Tabraham, P., et al (2005a) What causes the onset of psychosis? Schizophrenia Research, 79, 23-34.

Broome, M. R., Woolley, J. B., Johns, L. C., et al (2005b) Outreach and support in South London (OASIS): implementation of a clinical service for

M. R. BROOME, MRCPsych, OASIS, Section of Neuroimaging, Division of Psychological Medicine, Institute of Psychiatry, London, UK and Health Sciences Research Institute, Warwick Medical School, University of Warwick, Coventry, UK; L. C. JOHNS, DPhil, DClinPsy, I. VALLI, MD, J. B. WOOLLEY, MRCP, MRCPsych, P. TABRAHAM, DClinPsy, C. BRETT, PhD, OASIS, Section of Neuroimaging, Division of Psychological Medicine, Institute of Psychiatry, London, UK; L. VALMAGGIA, PhD, DClinPsy, OASIS, Section of Neuroimaging, Division of Psychological Medicine, Institute of Psychiatry, London, UK and Department of Psychiatry and Neuropsychology, Maastricht University, The Netherlands; E. PETERS, PhD, DClinPsy, P. A. GARETY, PhD, FBPsS, Department of Psychology, Institute of Psychiatry, London, UK; P. K. McGUIRE, PhD, FRCPsych, OASIS, Section of Neuroimaging, Division of Psychological Medicine, Institute of Psychiatry, London, UK

Correspondence: Dr Matthew Broome, Health Sciences Research Institute,Warwick Medical School, University of Warwick, Coventry CV4 7AI, UK. Email: m.broome@iop.kcl.ac.uk

prodromal psychosis and the at risk mental state. European Psychiatry, 20, 372-378

Broome, M. R., Matthiasson, P., Fusar-Poli, P., et a (2007) Neural correlates of executive function and working memory in the 'at-risk mental state'.

Colbert, S. M. \& Peters, E. R. (2002) Need for closure and jumping-to-conclusions in delusion-prone individuals. Journal of Nervous and Mental Disease, 190 $27-31$

Dudley, R. E., John, C. H., Young, A.W., et al (1997a) Normal and abnormal reasoning in people with delusions. British Journal of Clinical Psychology, 36 , 243-258.

Dudley, R. E., John, C. H., Young, A. W., et al (1997b) The effect of self-referent material on the reasoning of people with delusions. British Journal of Clinical Psychology, 36, 575-584

Freeman, D. (2007) Suspicious minds: the psychology of persecutory delusions. Clinical Psychology Review.

Freeman, D., Garety, P. A., Kuipers, E., et al (2002) A cognitive model of persecutory delusions. British Journal of Clinical Psychology, 4I, 331-347.

Freeston, M. H., Rheaume, J., Letarte, H., et al (1994) Why do people worry? Personality and Individual Differences, 17, 791-802.

Garety, P. A. \& Freeman, D. (1999) Cognitive approaches to delusions: A critical review of theories and evidence. British Journal of Clinical Psychology, 38, II3-154.

Garety, P. A., Freeman, D., Jolley, S., et al (2005) Reasoning, emotions, and delusional conviction in psychosis. Journal of Abnormal Psychology, II4, 373-384.

Garety, P. A., Bebbington, P., Fowler, D., et al (2007) Implications for neurobiological research of cognitive models of psychosis: a theoretical paper. Psychological Medicine, 37, 1377-1391.

Huq, S., Garety, P. \& Hemsley, D. (1988) Probabilistic judgements in deluded and non-deluded subjects. Quarterly Journal of Experimental Psychology A: Human Experimental Psychology, 40, 801-812.

Kapur, S. (2003) Psychosis as a state of aberrant salience: a framework linking biology, phenomenology, and pharmacology in schizophrenia. American Journal of Psychiatry, 160, 13-23.
Kay, S., Fiszbein, A. \& Opler, L. (1987) The positive and negative symptom scale (PANSS) for schizophrenia. Schizophenia Bulletin, 13, 261-276.

\section{Linney, Y. M., Peters, E. R. \& Ayton, P. (1998)}

Reasoning biases in delusion-prone individuals. British Journal of Clinical Psychology, 37, 285-302.

Miller, T. J., McGlashan, T. H., Rosen, J. L., et a (2003) Prodromal assessment with the structured interview for prodromal syndromes and the scale of prodromal symptoms: predictive validity, interrater reliability, and training to reliability. Schizophrenia Bulletin, 29, 703-715. (Erratum appears in Schizophrenia Bulletin 30, following 217 (2004)

Morey, R. A., Inan, S., Mitchell, T. V., et al (2005) Imaging frontostriatal function in ultra-high-risk, early, and chronic schizophrenia during executive processing. Archives of General Psychiatry, 62, 254-262.

Morrison, A. P., French, P., Walford, L., et al (2004) Cognitive therapy for the prevention of psychosis in people at ultra-high risk: randomised controlled trial. British Journal of Psychiatry, 185, 291-297.

Nelson, H. E. \& Willison, J. (199I) National Adult Reading Test (NART). nFER-Nelson.

Pantelis, C., Velakoulis, D., McGorry, P. D., et a (2003) Neuroanatomical abnormalities before and after onset of psychosis: a cross-sectional and longitudinal MRI comparison. Lancet, 36I, 28I-288.

Peters, E. R., Joseph, S. A. \& Garety, P. A. (1999) Measurement of delusional ideation in the normal population: Introducing the PDI (Peters et al Delusions Inventory). Schizophrenia Bulletin, 25, 553-576.

Van Dael, F., Versmissen, D., Janssen, I., et al (2006) Data gathering: biased in psychosis? Schizophrenia Bulletin, 32, 34I-35।

Wood, S. J., Pantelis, C., Proffitt, T., et al (2003) Spatial working memory ability is a marker of risk-forpsychosis. Psychological Medicine, 33, 1239-1247.

Yung, A. R., Phillips, L. J., McGorry, P. D., et al (1998) Prediction of psychosis - A step towards indicated prevention of schizophrenia. British Journal of Psychiatry 172, 14-20.

Yung, A. R., Phillips, L. J., Yuen, H. P., et al (2003)

Psychosis prediction: 12-month follow up of a high-risk ('prodromal') group. Schizophrenia Research, 60, 21-32. 